\title{
Anatomy of digital contact tracing: Role of age, transmission setting, adoption and case detection
}

\author{
Jesús A. Moreno López ${ }^{1}$, Beatriz Arregui García' ${ }^{1}$ Piotr Bentkowski' ${ }^{1}$ Livio Bioglio² ${ }^{2}$ Francesco Pinotti ${ }^{1}$, Pierre- \\ Yves Boëlle', Alain Barrat ${ }^{3,4}$, Vittoria Colizza', Chiara Poletto ${ }^{1 *}$ \\ ${ }^{1}$ INSERM, Sorbonne Université, Pierre Louis Institute of Epidemiology and Public Health, Paris, France. ${ }^{2}$ Department of Computer Science, University of Turin, Turin, Italy. \\ ${ }^{3}$ Aix Marseille Univ, Universite de Toulon, CNRS, CPT, Turing Center for Living Systems, Marseille, France. ${ }^{4}$ Tokyo Tech World Research Hub Initiative (WRHI), Tokyo \\ Institute of Technology, Tokyo, Japan \\ ${ }^{*}$ Corresponding author. Email: chiara.poletto@inserm.fr
}

The efficacy of digital contact tracing against COVID-19 epidemic is debated: smartphone penetration is limited in many countries, non-uniform across age groups, with low coverage among elderly, the most vulnerable to SARS-CoV-2. We developed an agent-based model to precise the impact of digital contact tracing and household isolation on COVID-19 transmission. The model, calibrated on French population, integrates demographic, contact-survey and epidemiological information to describe the risk factors for exposure and transmission of COVID-19. We explored realistic levels of case detection, app adoption, population immunity and transmissibility. Assuming a reproductive ratio $R=2.6$ and $50 \%$ detection of clinical cases, a $\sim 20 \%$ app adoption reduces peak incidence by $\sim 35 \%$. With $R=1.7,>30 \%$ app adoption lowers the epidemic to manageable levels. Higher coverage among adults, playing a central role in COVID19 transmission, yields an indirect benefit for elderly. These results may inform the inclusion of digital contact tracing within a COVID-19 response plan.

\section{Introduction}

Intervention measures aiming at preventing transmission have been key to control the first wave of the COVID-19 pandemic. Many countries have adopted lockdown and strong social distancing during periods of intense epidemic activity to suppress the epidemic and reduce hospital occupancy below saturation levels $(1,2)$. Due to their huge economic and societal costs these interventions can only be implemented for a limited amount of time. The building of population immunity has been slow (3-5), so that new waves are possible after temporary lockdowns and lifting of restrictions. Sustainable strategies are required to maintain the epidemic under control while enabling the close-tonormal functioning of the society. Widespread testing, case finding and isolation, contact-tracing, use of face masks and enhanced hygiene are believed to be crucial components of these strategies.

Contact-tracing aims to avoid transmission by isolating at an early stage only those individuals who are infectious or potentially infectious, in order to minimize the societal costs associated to isolation. Considerable resources are therefore directed at improving surveillance capacities to allow efficient and rapid investigation and isolation of cases and their contacts. To enhance tracing capacities, the use of digital technologies has been proposed, leveraging the wide-spread use of smartphones. Therefore, proximity-sensing applications have been designed and made available - e.g., in
Australia, France, Germany, Iceland, Italy, Switzerland - to automatically trace contacts, notify users about potential exposure to COVID-19 and invite them to isolate.

Empirical studies of the impact of these digital applications are however limited (6-8), and the utility of this intervention is debated. Some built-in features make it more efficient than manual contact tracing: it is automated, reducing the burden of manual contact tracing and limiting recall bias; it is faster, as information can be transmitted in real time. However, coverage is uneven. In particular, most children and elderly do not own a smartphone or are less familiar with digital technologies. The overall adoption of the app among smartphone owners will also be a limiting factor, as well as the fraction of cases actually triggering the alert to the contacts and the adherence to isolation of the app adopters who receive an alert.

These variables must be gauged in light of the risk factors for exposure and transmission driving the COVID-19 epidemic. First, individuals of different age contribute differently to the transmission dynamics of COVID-19. Younger individuals tend to have more contacts than adults or the elderly. On the other hand, a marked feature of COVID-19 is the strong age imbalance among cases $(9-13)$, that may be explained by both a reduced susceptibility $(9,10)$ and an increased rate of subclinical infections in children compared to adults $(10,11,13)$. As subclinical cases are harder to detect, this implies that identification of cases and of their contacts 
may be dependent on age. Second, SARS-CoV-2 transmission risk varies substantially by setting. Transmissions were registered predominantly in households, in specific workplaces and in the community (linked to shopping centers, meals, parties, sport classes, etc.) $(14,15)$. This is due, at least in part, to the higher risk of contagion of crowded and indoor environments (14-16). Notably, contacts occurring in the community are also the ones more affected by recall biases, thus more difficult to trace with manual contact tracing.

Several modelling studies have quantified the impact of contact tracing (17-26), with some of them addressing specific aspects of digital contact tracing (18-23). Still the interplay between age and setting heterogeneity in determining the efficacy of this intervention is largely unexplored. Here we provide a systematic exploration of the different variables at play. We considered France as a case study and integrated different sources of data to realistically describe the French population, in terms of its demography and social contact behavior. We accounted for the dynamics of contacts according to age and setting, and for the setting-specific risk of transmission. We used COVID-19 epidemiological characteristics for parametrization. We then modeled case detection and quarantining, isolation of their household contacts and digital contact tracing, under different hypotheses of potential reduction in transmissibility due to other effects (e.g., facemasks and increased hygiene). We quantified the impact of digital contact tracing on the whole population and on different population groups and settings, as a function of several variables such as the rate of app adoption, the probability of detection of clinical and subclinical cases, population immunity, compliance to isolation and transmission potential. Our results provide quantitative information regarding the impact of digital contact tracing within a broader response plan.

\section{Results \\ Dynamic multi-setting contact network}

We modeled the French population integrating available demographic and social-contact data. We collected population statistics on age, household size and composition (Fig. 1 A, B), workplace and school size, smartphone penetration (Fig. $1 \mathrm{E}$ ), and commuting fluxes. Then, by following standard approaches in the literature $(27,28)$ individuals were created insilico with a given age and assigned to a municipality, a household, and a workplace/school according to the statistics. Smartphones were assigned to individuals depending on their age according to available statistics on French users (Fig. 1 E) (29). Overall smartphone penetration was $64 \%$, that represents the upper bound limit of app adoption in the population - reached when $100 \%$ of individuals owning a smartphone download the app. This synthetic population reproduced the location statistics of individuals in different settings, yielding the basis of a multi-setting network of daily face-to-face contacts in household, school, workplace, community and transport (Fig. $1 \mathrm{H})(30-32)$. We parametrized the network from a social contact survey providing information on contacts by age and setting (33) (Fig. 1 C, D). As contacts may occur repeatedly, we associated an activation rate to each contact and sampled each day contacts based on their activation rate (Fig. $1 \mathrm{G}$ ). We imposed that $35 \%$ of the contacts registered during one day occur with daily frequency, as found in (33). Figure $1 \mathrm{~F}$ and I show that the features of the resulting daily contact network matched the data: the distribution of the number of contacts was right-skewed as the empirical one reported in (33) and the contact matrix showed age assortativity and the characteristic parent-children (offdiagonal) contact pattern. As a case study we restricted our study to a municipality with a population size of $\sim 100,000$ individuals (see Material and Methods and Supplementary Material for additional details).

\section{COVID-19 epidemic dynamics}

We modeled coronavirus transmission and outcome as shown in Fig. 2 A, B. Individuals could be susceptible, $S$, exposed, $E$, pre-symptomatic preceding subclinical infection, $I_{p, s c}$, pre-symptomatic preceding clinical infection, $I_{p, c}$, subclinically infectious, $I_{s c}$, clinically infectious, $I_{c}$, and recovered, $R$. Subclinical cases had symptoms that ranged from no symptoms to mild and continued their normal activity throughout the infectious period. Clinical cases had moderate to critical symptoms and stayed at home after the onset of symptoms $(11,13)$ - we did not consider hospitalization. Individuals in compartments $I_{p, s c}, I_{p, c}, I_{s c}, I_{c}$ transmitted the infection, with subclinical individuals characterized by a lower risk of transmission than clinical ones (see Material and Methods). We accounted for the heterogeneous susceptibility and clinical manifestation by age as parametrized from $(9,13)$ (Table 1$)$. In order to parametrize the infection's natural history, we combined evidence from epidemiological and viral shedding studies. We used 5.2 days for the incubation period (34), 2.3 days for the average length of the pre-symptomatic phase (35), and 7 days on average for the infectivity period after symptoms' onset (35).

We first simulated an uncontrolled epidemic assuming transmission levels corresponding to $R_{0}=3.1$, within the range of values estimated for COVID-19 in France at the early stage of the pandemic $(1,36)$. The generation time resulting from our model and parameters had mean value of 6.0 days (95\% CI $[2,17])$, in agreement with epidemiological estimates (11, 35, 37). Figure $2 \mathrm{C}$ and $\mathrm{D}$ show the repartition of cases among age groups and settings at the early stage and during the whole course of the epidemic. Age-specific infection probability was higher among young adults, while clinical 
infections were shifted toward older population with respect to the overall (clinical and subclinical) cases, as noted in previous observational and modelling works (10). The age profile changed in time with children infected later as the epidemic unfolded $(10,38)$. Transmissions occurred predominantly in household and workplaces followed by the community setting (14).

\section{Contact tracing}

We quantified the impact of combined household isolation and digital contact tracing considering the possible scenario of a new epidemic wave emerging after the release of strict lockdown measures in the country. We thus assumed some level of immunity to the virus - exploring a range from 0 to $15 \%$ of the population. We considered interventions based on the use of digital contact tracing, coupled with testing and isolation of clinical cases and households. $50 \%$ of individuals with clinical symptoms were assumed to get tested after consulting a doctor and to isolate if positive. Higher and lower percentages were also considered.

Case tracing was assumed to start when a case with clinical symptoms tested positive and was isolated, with an average delay of $\sim 1$ day. Household members were also invited to isolate - we assumed that $90 \%$ of them accepted to isolate and that their isolation occurred at the same time as the detected case. If the index case had the app installed, the contacts he/she registered in the previous $D=7$ days were notified and could decide to isolate with a compliance probability of $90 \%$ - lower values of compliance were also explored. Note that only contacts occurring between individuals who both use the app can be registered, so only app adopters can be notified. We explored several levels of app adoption in the population. In addition to the detection of clinical cases, we assumed that a proportion of subclinical cases was also identified. These may be cases with very mild, unspecific symptoms who decided to get tested as part of vulnerable groups (i.e., co-morbidity) or because highly exposed to the infection (health care professionals). We hypothesized this proportion to be small in the baseline scenario (5\%), and we later varied it up to $45 \%$. Isolated individuals resumed normal daily life if infection was not confirmed. We took 7 days as the time needed for being confirmed negative because multiple tests and some delay since the exposure are needed for a negative result to be reliable. Infected individuals got out of quarantine after 14 days unless they still have clinical symptoms after the time is passed. They may, however, decide to drop out from isolation each day with a probability of $2 \%$ if they don't have symptoms $(21,26)$.

Figure 3 summarizes the effect of the interventions. We compared the uncontrolled scenario ( $R=R_{0}=3.1$ ) with scenarios where the transmissibility is reduced due to the adoption of barrier measures ( $R$ down to 1.5). We also assumed
$10 \%$ of the population to be immune to the infection (36). Panels A-C shows the results for $R=2.6$ and $R=1.7$. With $R=2.6$ (Fig. $3 \mathrm{~A}, \mathrm{C}$ ), the relative reduction of peak incidence due to household isolation only would be $27 \%$. The inclusion of digital contact tracing would increase the relative reduction to $35 \%$ with $\sim 20 \%$ app adoption, and to $66 \%$ with $\sim 60 \%$ app adoption - i.e., $90 \%$ of individuals owning a smartphone use the app. This corresponds to an additional mitigation effect ranging from $30 \%$ to $144 \%$ provided by contact tracing compared to household isolation only. With $R=1.7$ (Fig. $3 \mathrm{~B}$, $\mathrm{C}$ ), we find that $\sim 20 \%$ app adoption would reduce the peak incidence by $45 \%$ (additional mitigation effect of $25 \%$ ), while the reduction would reach $89 \%$ in a scenario of $\sim 60 \%$ app adoption (additional mitigation effect of $147 \%$ ). According to the projections in Ref. (1), intensive care units occupation would remain below the saturation level with incidence below $0.4 / 1000$ hab. In the scenario with $R=1.7$, this would be reached with app adoption greater than $\sim 30 \%$ (grey dashed line in Fig. 3 B). Stronger reductions could be obtained with more efficient detection of clinical cases (Fig. 3 E, H, obtained with $R=2.6$ ) and of subclinical ones (Fig. $3 \mathrm{~L}$, $R=2.6$ ). The relative reduction in peak incidence produced by $\sim 20 \%$ app adoption would be $47 \%$ with an $80 \%$ detection rate of clinical cases, compared to the $35 \%$ relative reduction obtained with $50 \%$ detection rate. Results show similar trends across different levels of population immunity, with higher relative impacts predicted for low immunity (Fig. $3 \mathrm{~F}$, I, $R=2.6$ ). Compliance to isolation of household contacts had an appreciable effect at low app adoption (Fig. $3 \mathrm{~K}$, $R=2.6$ ). $50 \%$ compliance would reduce peak incidence of $19 \%$, compared to $27 \%$ reduction for $90 \%$ compliance, in the case of household isolation only. Compliance of notified contacts to isolation, instead, has a larger effect on peak incidence only when app adoption is high, as expected. For example, if the app was adopted by $60 \%$ of individuals the reduction in peak incidence would pass from $55 \%$ to $66 \%$ if compliance changed from $50 \%$ to $90 \%$ (Fig. $3 \mathrm{~J}, R=2.6$ ).

We analyzed the simulation outputs to characterize index cases and their contacts and relate this to the reduction in number of cases by age and setting. We found that adults represented the majority of index cases (Fig. 4 D), while their household contacts were mostly children. The app registered mostly contacts with adults, and the tracked contacts were occurring predominantly in workplaces and in the community (Fig. $4 \mathrm{~A}$ ). This results in a heterogeneous reduction in transmission (TRR) by setting and age group. Household isolation reduced transmission in all settings, with the smallest effect in workplaces (Fig. 4 B). Digital contact tracing has instead a high TRR at work, in the community and in transports (Fig. $4 \mathrm{C}$ ). Household isolation reached mostly children ( $<15$ years old) and the elderly (especially the $75+$ group) with the smallest effect in the 15-59 years old (Fig. 4 E). Adopting 
digital contact tracing led to an increased TRR with age, even among the oldest age range (Fig. $4 \mathrm{~F}$ ). This result shows the indirect effect of digital tracing: due to the central role of adults in the transmission of SARS-CoV-2 toward all agegroups, avoiding adult infections led to less transmission to the elderly. We also tested the case in which elderly people (70+) owning a smartphone did not install the app at all, assuming they are less familiar with digital technologies, and we found no appreciable effect. These results and additional details are provided in the Supplementary Material.

\section{Traced and isolated individuals}

Feasibility of contact tracing depends on the number of traced contacts who require assistance and virological tests. In a scenario with high detection rate (80\%), we found that for each detected case 1.5 contacts were identified on average through household isolation but up to 7.5 with app adoption at $57 \%$, for $R=2.6$ and 10 for $R=1.7$ (Fig. 5 D). This number was however subject to fluctuations (Fig. $5 \mathrm{~A}$ ). Overall, the maximal fraction of the population quarantined at any given time was $\sim 50$ per 1000 habitants in a scenario with $R=2.6$, and was between $\sim 1$ and $\sim 4.5$ per 1000 habitants when $R=1.7$ (Fig. $5 \mathrm{~B}$ and $\mathrm{E}$ ). The latter case corresponded to the situation in which high levels of app adoption were able to strongly reduce spreading, thus the proportion of isolated individuals declined in time, signaling the success of quarantining in preventing the propagation of the infection. A total of 30 per 1000 habitants were isolated in a scenario with $R=1.7$, assuming high app adoption. At $R=2.6,1030$ per 1000 habitants were isolated at the end of the epidemic meaning that certain individuals were isolated more than once. In all scenarios, the increase of app adoption inevitably determined an increase in the proportion of people that were unnecessarily isolated, i.e., of individuals that were not infected but still isolated (Fig. 5C, F): this proportion increased from $61 \%$ to $84 \%$ with the increase of app adoption from $0 \%$ to $57 \%$ (note that the case of $0 \%$ app adoption implies that $61 \%$ of individuals who were isolated through household isolation were not infected). These numbers were similar for the two tested values of $R=1.7$ and 2.6 (Fig. $5 \mathrm{C}$ and F).

\section{Discussion}

Quantifying the impact of digital contact tracing is essential to envision this strategy within a wider response plan against the COVID-19 epidemic. We modeled this intervention together with household isolation assuming a 50\% detection of clinical cases. In a scenario of high transmissibility $(R=2.6$ ), we found that household isolation by itself would produce a reduction in peak incidence of $27 \%$, while the inclusion of digital contact tracing could increase this effect by $30 \%$ for a reasonably achievable app adoption ( $\sim 20 \%$ of the population), and by $144 \%$ for a large-scale app adoption ( 60\%). At a moderate transmissibility level $(R=1.7)$, the app would substantially damp transmission (36\% to $89 \%$ peak incidence reduction for increasing app adoption), bringing the epidemic to manageable levels if adopted by $32 \%$ of the population or more. Importantly, the app-based tracing and household isolation have different effects across settings, the first intervention efficiently preventing transmissions at work that are not well targeted by the second. Moreover, appbased contact tracing also yields a protection for the elderly despite the lower penetration of smartphones in this age category.

Lockdown and social distancing have been effective in reducing transmission in the first epidemic wave in many countries. However, their huge societal and economic costs made their prolonged implementation impossible. Phasing out lockdown occurred at the beginning of the summer in Europe, with high temperatures, increased ventilation and outdoor activities helping reducing the risk of contagion (16). The relaxation of almost all restrictive measures, the start of activities in the fall and the cold season accelerated transmission, reaching a point in which strict non-pharmaceutical interventions were again necessary to curb the epidemic increase. At the time of writing, national or local lockdowns were restored in several countries in Europe (39). This highlights the need for exit strategies based on sustainable nonpharmaceutical interventions, able to suppress COVID-19 spread while having limited impact on the economy and on individuals' daily life (1).

Many countries have increased their capacity to detect cases and track their contacts. In France, thousands of transmission clusters have been identified and controlled since the end of the first lockdown period (40). Under-detection of cases was however estimated over summer, and the system was predicted to deteriorate rapidly for increasing epidemic activity (41). The automated tracking of contacts could then provide an important complementary tool. Here we found that digital contact tracing could reduce attack rate and peak incidence, in agreement with previous works $(18,19,26)$. The impact of the measure would depend on population immunity, thus geographical heterogeneities should be expected, as regions were differentially hit by the first wave of the epidemic (4). On the other hand, app adoption as well may be higher in these areas because of risk aversion behavior (42). Also, higher participation rates may be expected in dense urban areas to protect from exposure from random encounters (e.g., in public transports).

Under realistic hypotheses, the intervention would not be able alone to bring the epidemic under control in a scenario where transmission is high $(18,19,26)$, mainly due to the strong role of asymptomatic transmission in fueling the epidemic $(11,12,43)$. We explored different values of the reproductive number $R$, to effectively account for non- 
pharmaceutical measures mitigating the epidemic and for the adoption of preventive measures substantially hindering SARS-COV-2 transmission. We found that a reduction of the epidemic to a manageable level would be possible with a moderate $R$ (e.g., $R=1.7$ explored here).

Improved case finding is the first step toward a successful contact-tracing intervention. We found that the increase in detection of clinical cases substantially reduced peak incidence and improved the efficacy of contact tracing. Many countries progressively increased testing capacity (41) and lifted restrictions on access to testing (44). Easy access to testing is essential to detect cases, because of the substantial fraction of subclinical cases and the similarity of COVID-19 clinical presentation to the one of other respiratory infections. In the period from September to November 2020, the French network of virological surveillance run by general practitioners reported that only $22.7 \%$ of Acute Respiratory Infections were caused by SARS-CoV2, against $46.5 \%$ attributed to rhinovirus (45). Given that the majority of cases do not require hospitalization, case detection effectiveness is also influenced by the consultation rate. This has been estimated to be around $\sim 30 \%$ with peaks at $\sim 45 \%$ by the participatory surveillance platform covidnet.fr $(40,41)$. Increased population awareness is thus essential for the efficient monitoring of the epidemic and its containment through contact tracing.

Little information is available on compliance to isolation. Low compliance to isolation was reported in the UK and in a university campus in the US $(46,47)$. However, this may vary greatly according to cultural, socioeconomic and demographic context. Due to a self-selection bias, individuals who decided to download and use the mobile application may be more akin to follow the recommendation and isolate if they receive a notification. Step-by-step recommendations provided by the app can further help in increasing compliance. Strengthened communication and compensations (such as paid work leave, loss-of-income payments for self-employed professionals, medical school-absence certificates) should be implemented to increase the acceptability of isolation (48).

App adoption remains the key factor determining the efficacy of digital contact tracing. Adoption levels were initially low (<5\%) in many countries (e.g., Italy, France), increasing later as the second wave was rising, likely due to increased concerns of the population. As of November 2020, $17 \%$ and $13 \%$ of the population had downloaded the app in Italy and France, respectively $(49,50)$. Higher levels were observed, e.g., in Australia (6 millions download, $25 \%$ of the population) (51) and Iceland ( 150 thousand, 38\%) (52). Importantly, official figures may overestimate real adoption levels, since many individuals may download the application without using it. In France this proportion was $60 \%$ among university students (53). Individuals may be more inclined to use the app if they perceive a direct and immediate benefit from its use. This may be implemented through, e.g., easy access to testing in case they are notified as contacts and assistance by public health professionals. Moreover, even if the application preserves users' privacy and can be downloaded on a voluntary basis in many countries, increased transparency and ethical debate remain essential to reassure the population about data treatment (53-55).

The results presented here are based on an agent-based model that describes age-specific risk factors for exposure and transmission: contact rates, contacts by location, susceptibility to the virus, probability of being detected and rate of app adoption. The interplay between these features has a profound impact on COVID-19 spread and affects the efficacy of household isolation and digital contact tracing. To account for contact heterogeneities we used statistics on population demography, combined with social contact surveys to build a multi-setting contact network, similarly to previous works $(17,21,26,30-32)$. The network is also dynamic in time as it captures the repetition of a certain number of contacts (e.g., relationships) and the occurrence of random encounters. Social contact data provide an invaluable information source to study the current COVID-19 outbreak $(1,36)$. Previous projections on the impact of contact tracing rely on a similar approach in some cases $(17,24,26)$. Other works make use of high resolution data $(18,19,22)$, that are more reliable than contact surveys, but are restricted to specific settings or population groups. Despite the difference in the data source and approach, the results of these studies are consistent and in agreement with our work on the overall impact of the intervention.

We modeled age-specific epidemiological characteristics based on available knowledge in the literature. Children are less impacted by the COVID-19 epidemic (9-13). This may be explained by reduced susceptibility and severity, with accumulating evidence that both effects are acting simultaneously (10). The strength of these effects is still debated and the infection risk for children should not be minimized. However, these differences have implications for digital contact tracing. Indeed, it is precisely in the group that plays a central role in transmission and where cases are more likely symptomatic (i.e., adults) that the app coverage is already the highest. Our model shows that taken together, these characteristics reinforce the impact of digital tracing and provides indirect protection in the elderly population. This occurs even if no adoption is registered in the elderly population.

Our study is affected by limitations. First, we analyzed the effect of digital contact tracing on COVID-19 incidence in the general population. Crucial information for public health authorities would be to quantify the effect in time of these measures on hospitalizations. This would require to couple 
our model for COVID-19 transmission in the general population with a model describing disease severity and within-hospital patient trajectories $(17,21,26)$. Second, the model does not account for transmission in nursing homes. This setting is where the majority of transmissions among elderly occurred. At the same time, however, the response to the COVID-19 epidemic in this setting relies mostly on routine screening of symptoms and frequent testing of residents, together with face masks and strict hygiene rules for visitors. Third, results may be conservative as clustering effects and large fluctuations in the number of contacts per person (56) are only partially captured by the model thanks to the repetition of contacts, but effects may be larger in real contact patterns. This also includes crowding events playing an important role in the transmission dynamics (15). Overall behaviors obtained with our synthetic network of contacts are however compatible with findings obtained with real contact data (18). In a future work the description of temporal and topological properties of contacts in workplaces, schools and community could be improved by using modeling frameworks informed by detailed contact data, that has become available for specific settings (57-59). For this purpose, frameworks such as hidden variable models or other recent dynamical models for social networks could be employed (60-62). Fourth, other assumptions may be instead optimistic, regarding the probability of detection of index cases, and compliance to isolation, for example. Few data are available to inform these parameters that may also vary over time (depending on the epidemic context and increased population fatigue) and across countries (depending on cultural aspects and regulations in place). While we explored a range of parameter values, more detailed information will be needed to contextualize our approach to a specific epidemic situation in a given country.

\section{Material and Methods Synthetic population}

The model simulates the population of Metropolitan France representing individual inhabitants. This approach is similar to studies done previously e.g., for Italy (27) and for USA (28). The French synthetic population is based on the National Institute of Statistics and Economic Studies (INSEE) censuses. Individuals were assigned to municipalities according to the administrative borders. The number of households and the age structure of their inhabitants, sizes of schools and workplaces, fluxes of commuters between municipalities also followed the distribution of these statistics found in the INSEE data. Population size was kept constant through a simulation as we aimed at simulating one season of the epidemic.

To generate the population, we defined several statistics derived from INSEE publicly available data:

The list of municipalities ("les communes de France") of
Metropolitan France (2015) with each municipality described by its INSEE code, population size, number of schools of six different levels (from kindergarten to university), number of workplaces in given size categories (0-9, 10-49, 50-99, 100499, 500-999 and over 1000 employees) (Populations légales 2017, INSEE, $\quad$ https://www.insee.fr/fr/statistiques $/ 4265429$ ? sommaire $=4265511$ ).

Statistics regarding the percentage of people in given age groups enrolled in each of six school levels, employed and unemployed (Bilan démographique 2010, INSEE, https://www.insee.fr/fr/statistiques/1280950).

The age pyramid for France as the population fractions of individuals of a given age (Bilan démographique 2010, INSEE, https://www.insee.fr/fr/statistiques/1280950).

The number of people commuting to work between each pair of municipalities (Mobilités professionnelles en 2016: déplacements domicile - lieu de travail, INSEE, https://www.insee.fr/fr/statistiques/4171554).

The number of people commuting to school between each pair of municipalities (Mobilités scolaires en 2015: déplacements domicile - lieu d'études, INSEE, https://www.insee.fr/fr/statistiques/3566470).

The probability distributions of sizes of households in France (Couples - Familles - Ménages en 2010. INSEE, https://www.insee.fr/fr/statistiques/2044286/?geo=COM34150.)

The probability of individuals belonging to a particular age class, given their role in the household: child of a couple, child of a single adult, adult in a couple without children, adult in a couple with children (Couples - Familles - Ménages en 2010. INSEE, https://www.insee.fr/fr/statistiques/2044286/?geo=COM-34150).

With the above statistics, the synthetic population was generated in the following steps:

1.Initialization of all the municipalities with an appropriate number of schools of each type and workplaces of given sizes.

2.Creation of schools in each municipality according to given statistics.

3.Creation of workplaces in each municipality according to given statistics.

4.Definition of the commuter fluxes between municipalities.

Each municipality has a defined number of inhabitants and individuals are created (one by one) until this number is reached. Each individual was assigned an age, a school or a workplace (or is assigned to stay at home) according to probability distributions derived from the data mentioned above.

The numbers of households within each municipality were not defined explicitly, but depended on the number of individuals. The municipal population size and statistics regarding family demographics constrain the number of 
households. Additional details on the algorithm for the population reconstruction are provided in the Supplementary Material.

\section{Face-to-face contact network}

The synthetic population encodes information on the school, workplace, household and community each individual belongs to. We used this information to extract a dynamic network representing daily face-to-face contacts. We parametrized this network based on contacts' statistics for the French population (33).

First, we generated a time aggregated network representing all contacts that can potentially occur - we will call this the acquaintance network, with some abuse of language since it includes also sporadic contacts. Second, to each contact we assigned a daily rate of activation. Then, in the course of the simulation we sampled contacts each day based on their rate.

The acquaintance network has five distinct layers representing contacts in household (layer $H$ ), workplace (layer $W$ ), school (layer $S$ ), community (layer $C$ ) and transports (layer $T$ ). The household layer is formed by a collection of complete networks linking individuals in the same household. The $W, S, C$, and $T$ layers are formed by collections of Erdős-Rényi networks generated in each location $i$, with average degree $\chi_{i}$. A location can be a workplace ( $W$ layer), a school ( $S$ layer) and a municipality ( $C$ and $T$ layers). $\chi_{i}$ is extracted at random for each place and depends on the type and size of the location. In particular, when the size of a location is small we assume that each individual enters in contact with all the others frequenting the same place. As the size increases the number of contacts saturates.

Once the acquaintance network was built a daily activation rate $x$ was assigned to each link according to a cumulative distribution that depends on the layer $s$. For simplicity we assumed this distribution to be the same for $s=W, S, C$, while we allowed it to be different in household (where contacts are more frequent) and in transports (where contacts are sporadic). Parameters were tuned based on average daily number of contacts, proportion of contacts by setting, and contact frequency as provided in (33) (Fig. $1 \mathrm{C}$ and D). Additional details on the network reconstruction and parametrization are provided in the Supplementary Material.

\section{Transmission model}

We defined a minimal model of COVID-19 spread in the general population that accounts for two levels of symptoms: none to mild (subclinical cases, $I_{s c}$ ), and moderate to severe (clinical cases, $I_{c}$ ). We assumed that clinical cases stay at home after developing symptoms. Susceptible individuals, if in contact with infectious ones, may get infected and enter the exposed compartment ( $E)$. After an average latency period $\epsilon^{-1}$ they become infectious, developing a subclinical infection with probability $p_{s c}^{A}$ and a clinical infection otherwise. From $E$, before entering in either $I_{s c}$ or $I_{c}$, individuals enter first a prodromal phase (either $I_{p, s c}$ or $I_{p, c}$ ), that lasts on average $\mu_{p}^{-1}$ days and where individuals do not show any sign of illness, despite being already infectious. Contact-tracing, population-screening and modelling studies provide evidence that infectivity is related to the level of symptoms, with less severely hit individuals being also less infectious (11, 43). Therefore, we assumed that subclinical cases, $I_{p, s c}$ and $I_{s c}$ have a reduced transmissibility compared to $I_{p, c}$ and $I_{c}$. This is modulated by the scaling factor $\beta_{I}$. We neglected hospitalization and death and assumed that with rate $\mu$ infected individuals become recovered.

The impact of COVID-19 is heterogeneous across age groups $(9-13)$. This may be driven by differences in susceptibility (9), differences in clinical manifestation $(11,13)$ or both (10). We considered here both effects in agreement with recent modelling estimates (10). Susceptibility by age, $\sigma_{A}$, was parametrized from (9), while clinical manifestation, $p_{s c}^{A}$, was parametrized from a large-scale descriptive study of the COVID19 outbreak in Italy (13).

Transition rates are summarized in Fig. 2 B, and parameters and their values are listed in Table 1 . The incubation period was estimated to be around 5.2 days from an early analysis of 425 patients in Wuhan (34). COVID-19 transmission potential varies across settings, populations and social contexts (14-16). In particular, indoor places were found to increase the odds of contagion 18.7 times compared to an open-air environment (16). In our model we assumed that all contacts at work, school and transport occur indoor and have the same transmission risk $\left(\omega_{S}\right)$. In the contact survey of Béraud et al. (33), $46 \%$ of contacts in the community were occurring outdoors. Combining this information with the 18.7 indoor vs. outdoor risk ratio leads to a $60 \%$ relative risk of community contacts with respect to workplace/school/transport contacts. Contacts within households are generally associated to a higher risk with respect to other settings, because they last longer and there is a higher risk of environmental transmission. We assumed that the transmission risk associated with them is twice the one in workplace/school/transport. For the basic reproductive ratio of COVID-19 we took $R_{0} \sim 3.1(1,36)$. We also explored lower levels of transmission potential, i.e., reproductive ratios $R$ down to 1.5 , to effectively account for behavioral changes and adoption of barrier measures. Our definition of $R$ does not integrate population immunity. We explicitly indicate the initial level of population immunity to disentangle the relative 
role of the two quantities.

\section{Modelling contact tracing}

Self-isolation and isolation of household contacts

Self-isolation and isolation of household contacts was modeled according to following rules:

As an individual shows clinical symptoms, s/he is detected with probability $p_{d, c}$ (baseline value 50\%, additional explored values $30 \%$ and $80 \%$ ). If detected, case confirmation, isolation and contacts' isolation occur with rate $r_{d, c}=0.9$ upon symptoms onset.

Subclinical individuals are also detected with probability $p_{d, s c}$ (baseline value 5\%, additional explored values $25 \%$ and $45 \%$ ), and rate $r_{d, s c}=0.5$.

The individual's family members are isolated with probability $p_{c, h}=0.9$ (0.5 and 0.7 were also explored).

We assume that contacts are tested and the follow up guarantees that all individuals who got infected prior to isolation are detected. Thus, contacts that are negative (either susceptible or recovered at the time of isolation) terminate their isolation after 7 days. The index-case and the positive contacts are isolated for 14 days. Contacts with no clinical symptoms have a daily probability $p_{d r o p}=0.02$ to drop-out from isolation.

For both the case and the contacts, isolation is implemented by assuming no contacts outside the household and contacts within a household having an associated transmission risk (i.e., the weight $\omega_{H}$ ) reduced by a factor $\imath=0.5$.

\section{Digital contact tracing}

We assumed that contact tracing is adopted in combination with self-isolation and isolation of household members. Therefore, we added the following rules to the ones outlined above:

At the beginning of the simulation, a smartphone is assigned to individuals with probability $p_{s m}^{A}$, based on the statistics of smartphone penetration (0\% for [0,11], $86 \%$ for $[12,17], 98 \%$ for $[18,24], 95 \%$ for $[25,39], 80 \%$ for $[40,59], 62 \%$ for $[60,69], 44 \%$ for $70+)(29)$.

Each individual with a smartphone has a probability $p_{a}$ to download the app (we explored values between 0 and 0.9 ).

Only contacts occurring between individuals with a smartphone and the app are traced.

If the individual owns a smartphone and downloaded the app the contacts that $\mathrm{s} /$ he has traced in the period since $D=7$ days before his/her detection are isolated with probability $p_{c, a}=0.9$ (0.5 and 0.7 were also tested).

We assume contacts are tested and the follow up guarantees that all individuals who got infected prior to isolation are detected. Thus, contacts that are negative (either susceptible or recovered at the time of isolation) terminate their isolation after 7 days. The index-case and the positive contacts are isolated for 14 days. Contacts with no clinical symptoms have a daily probability $p_{\text {drop }}=0.02$ to drop out from isolation.

For both the case and the contacts, isolation is implemented by assuming no contacts outside the household and contacts within a household having an associated transmission risk (i.e., the weight $\omega_{H}$ ) reduced by a factor $l=0.5$.

\section{REFERENCES AND NOTES}

1. L. Di Domenico, G. Pullano, C. E. Sabbatini, P.-Y. Boëlle, V. Colizza, Impact of lockdown on COVID-19 epidemic in Île-de-France and possible exit strategies. BMC Med. 18, 240 (2020). doi:10.1186/s12916-020-01698-4 Medline

2. S. Flaxman, S. Mishra, A. Gandy, H. J. T. Unwin, T. A. Mellan, H. Coupland, C. Whittaker, H. Zhu, T. Berah, J. W. Eaton, M. Monod, A. C. Ghani, C. A. Donnelly, S. Riley, M. A. C. Vollmer, N. M. Ferguson, L. C. Okell, S. Bhatt; Imperial College COVID-19 Response Team, Estimating the effects of non-pharmaceutical interventions on COVID-19 in Europe. Nature 584, 257-261 (2020). Medline

3. M. Pollán, B. Pérez-Gómez, R. Pastor-Barriuso, J. Oteo, M. A. Hernán, M. PérezOlmeda, J. L. Sanmartín, A. Fernández-García, I. Cruz, N. F. de Larrea, M. Molina, F. Rodríguez-Cabrera, M. Martín, P. Merino-Amador, J. L. Paniagua, J. F. MuñozMontalvo, F. Blanco, R. Yotti, F. Blanco, R. G. Fernández, M. Martín, S. M. Navarro, M. Molina, J. F. Muñoz-Montalvo, M. S. Hernández, J. L. Sanmartín, M. CuencaEstrella, R. Yotti, J. L. Paniagua, N. F. de Larrea, P. Fernández-Navarro, R. PastorBarriuso, B. Pérez-Gómez, M. Pollán, A. Avellón, G. Fedele, A. Fernández-García, J. O. Iglesias, M. T. P. Olmeda, I. Cruz, M. E. F. Martinez, F. D. Rodríguez-Cabrera, M. A. Hernán, S. P. Fernández, J. M. R. Aguirre, J. M. N. Marí, B. P. Borrás, A. B. P. Jiménez, M. Rodríguez-lglesias, A. M. C. Gascón, M. L. L. Alcaine, I. D. Suárez, O. S. Álvarez, M. R. Pérez, M. C. Sanchís, C. J. V. Gomila, L. C. Saladrigas, A. H. Fernández, A. Oliver, E. C. Feliciano, M. N. G. Quintana, J. M. B. Fernández, M. A. H. Betancor, M. H. Febles, L. M. Martín, L.-M. L. López, T. U. Miota, I. D. B. Población, M. S. C. Pérez, M. N. V. Fernández, T. M. Enríquez, M. V. Arranz, M. D.G. González, I. Fernández-Natal, G. M. Lobón, J. L. M. Bellido, P. Ciruela, A. M. Casals, Prevalence of SARS-CoV-2 in Spain (ENE-COVID): A nationwide, population-based seroepidemiological study. Lancet 0, (2020). 10.1016/SO1406736(20)31483-5

4. F. Carrat, X. de Lamballerie, D. Rahib, H. Blanche, N. Lapidus, F. Artaud, S. Kab, A. Renuy, F. S. de Edelenyi, L. Meyer, N. Lydie, M.-A. Charles, P.-Y. Ancel, F. Jusot, A. Rouquette, S. Priet, P. M. S. Villaroel, T. Fourie, C. Lusivika-Nzinga, J. Nicol, S. Legot, N. Druesne-Pecollo, Y. Essedik, C. Lai, J.-M. Gagliolo, J.-F. Deleuze, N. Bajos, G. Severi, M. Touvier, M. Zins, Seroprevalence of SARS-CoV-2 among adults in three regions of France following the lockdown and associated risk factors: a multicohort study. medRxiv (2020), 10.1101/2020.09.16.20195693

5. S. L. Vu, G. Jones, F. Anna, T. Rose, J.-B. Richard, S. Bernard-Stoecklin, S. Goyard, C. Demeret, O. Helynck, C. Robin, V. Monnet, L. P. de Facci, M.-N. Ungeheuer, L. Léon, Y. Guillois, L. Filleul, P. Charneau, D. Lévy-Bruhl, S. van der Werf, H. Noel, Prevalence of SARS-CoV-2 antibodies in France: results from nationwide serological surveillance. medRxiv (2020), doi:10.1101/2020.10.20.20213116.

6. M. Kendall, L. Milsom, L. Abeler-Dörner, C. Wymant, L. Ferretti, M. Briers, C. Holmes, D. Bonsall, J. Abeler, C. Fraser, Epidemiological changes on the Isle of Wight after the launch of the NHS Test and Trace programme: A preliminary analysis. The Lancet Digital Health 2, e658-e666 (2020). 10.1016/S2589-7500(20)30241-7 Medline

7. I. Braithwaite, T. Callender, M. Bullock, R. W. Aldridge, Automated and partly automated contact tracing: A systematic review to inform the control of COVID19. The Lancet Digital Health 2, e607-e621 (2020). doi:10.1016/S25897500(20)30184-9 Medline

8. M. Salathé, C. Althaus, N. Anderegg, D. Antonioli, T. Ballouz, E. Bugnon, S. Čapkun, D. Jackson, S.-I. Kim, J. Larus, N. Low, W. Lueks, D. Menges, C. Moullet, M. Payer, J. Riou, T. Stadler, C. Troncoso, E. Vayena, V. von Wyl, Early evidence of effectiveness of digital contact tracing for SARS-CoV-2 in Switzerland. Swiss Med. Wkly. 150, w20457 (2020). 10.4414/smw.2020.20457 Medline 
9. J. Zhang, M. Litvinova, Y. Liang, Y. Wang, W. Wang, S. Zhao, Q. Wu, S. Merler, C. Viboud, A. Vespignani, M. Ajelli, H. Yu, Changes in contact patterns shape the dynamics of the COVID-19 outbreak in China. Science 368, 1481-1486 (2020).

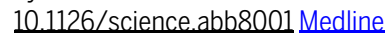

10. N. G. Davies, P. Klepac, Y. Liu, K. Prem, M. Jit, R. M. Eggo; CMMID COVID-19 working group, Age-dependent effects in the transmission and control of COVID19 epidemics. Nat. Med. 26, 1205-1211 (2020). doi:10.1038/s41591-020-0962-9 Medline

11. Q. Bi, Y. Wu, S. Mei, C. Ye, X. Zou, Z. Zhang, X. Liu, L. Wei, S. A. Truelove, T. Zhang, W. Gao, C. Cheng, X. Tang, X. Wu, Y. Wu, B. Sun, S. Huang, Y. Sun, J. Zhang, T. Ma, J. Lessler, T. Feng, Epidemiology and transmission of COVID-19 in 391 cases and 1286 of their close contacts in Shenzhen, China: A retrospective cohort study. Lancet Infect. Dis. 20, 911-919 (2020). doi:10.1016/S1473-3099(20)30287-5 Medline

12. E. Lavezzo, E. Franchin, C. Ciavarella, G. Cuomo-Dannenburg, L. Barzon, C. Del Vecchio, L. Rossi, R. Manganelli, A. Loregian, N. Navarin, D. Abate, M. Sciro, S. Merigliano, E. De Canale, M. C. Vanuzzo, V. Besutti, F. Saluzzo, F. Onelia, M. Pacenti, S. G. Parisi, G. Carretta, D. Donato, L. Flor, S. Cocchio, G. Masi, A. Sperduti, L. Cattarino, R. Salvador, M. Nicoletti, F. Caldart, G. Castelli, E. Nieddu, B. Labella, L. Fava, M. Drigo, K. A. M. Gaythorpe, A. R. Brazzale, S. Toppo, M. Trevisan, V. Baldo, C. A. Donnelly, N. M. Ferguson, I. Dorigatti, A. Crisanti; Imperial College COVID-19 Response Team; Imperial College COVID-19 Response Team, Suppression of a SARS-CoV-2 outbreak in the Italian municipality of Vo'. Nature 584, 425-429 (2020). doi:10.1038/s41586-020-2488-1 Medline

13. F. Riccardo, M. Ajelli, X. D. Andrianou, A. Bella, M. D. Manso, M. Fabiani, S. Bellino, S. Boros, A. M. Urdiales, V. Marziano, M. C. Rota, A. Filia, F. D’Ancona, A. Siddu, O. Punzo, F. Trentini, G. Guzzetta, P. Poletti, P. Stefanelli, M. R. Castrucci, A. Ciervo, C. D. Benedetto, M. Tallon, A. Piccioli, S. Brusaferro, G. Rezza, S. Merler, P. Pezzotti, the C.-19 working Group, Epidemiological characteristics of COVID-19 cases and estimates of the reproductive numbers 1 month into the epidemic, Italy, 28 January to 31 March 2020. Euro Surveill. 25, 2000790 (2020). doi:10.2807/1560-7917.ES.2020.25.49.2000790

14. Q. J. Leclerc, N. M. Fuller, L. E. Knight, S. Funk, G. M. Knight; CMMID COVID-19 Working Group, What settings have been linked to SARS-CoV-2 transmission clusters? Wellcome Open Res. 5, 83 (2020). doi:10.12688/wellcomeopenres.15889.2 Medline

15. B. M. Althouse, E. A. Wenger, J. C. Miller, S. V. Scarpino, A. Allard, L. HébertDufresne, H. Hu, Superspreading events in the transmission dynamics of SARSCoV-2: Opportunities for interventions and control. PLOS Biol. 18, e3000897 (2020). doi:10.1371/journal.pbio.3000897 Medline

16. H. Nishiura, H. Oshitani, T. Kobayashi, T. Saito, T. Sunagawa, T. Matsui, T. Wakita, M. C.-19 R. Team, M. Suzuki, Closed environments facilitate secondary transmission of coronavirus disease 2019 (COVID-19). medRxiv (2020), doi:10.1101/2020.02.28.20029272.

17. A. Aleta, D. Martín-Corral, A. Pastore Y Piontti, M. Ajelli, M. Litvinova, M. Chinazzi, N. E. Dean, M. E. Halloran, I. M. Longini Jr., S. Merler, A. Pentland, A. Vespignani, E. Moro, Y. Moreno, Modelling the impact of testing, contact tracing and household quarantine on second waves of COVID-19. Nat. Hum. Behav. 4, $964-$ 971 (2020). doi:10.1038/s41562-020-0931-9 Medline

18. G. Cencetti, G. Santin, A. Longa, E. Pigani, A. Barrat, C. Cattuto, S. Lehmann, B. Lepri, Using real-world contact networks to quantify the effectiveness of digital contact tracing and isolation strategies for Covid-19 pandemic. medRxiv (2020), doi:10.1101/2020.05.29.20115915.

19. A. J. Kucharski, P. Klepac, A. J. K. Conlan, S. M. Kissler, M. L. Tang, H. Fry, J. R. Gog, W. J. Edmunds, J. C. Emery, G. Medley, J. D. Munday, T. W. Russell, Q. J. Leclerc, C. Diamond, S. R. Procter, A. Gimma, F. Y. Sun, H. P. Gibbs, A. Rosello, K. van Zandvoort, S. Hué, S. R. Meakin, A. K. Deol, G. Knight, T. Jombart, A. M. Foss, N. I. Bosse, K. E. Atkins, B. J. Quilty, R. Lowe, K. Prem, S. Flasche, C. A. B. Pearson, R. M. G. J. Houben, E. S. Nightingale, A. Endo, D. C. Tully, Y. Liu, J. Villabona-Arenas, K. O'Reilly, S. Funk, R. M. Eggo, M. Jit, E. M. Rees, J. Hellewell, S. Clifford, C. I. Jarvis, S. Abbott, M. Auzenbergs, N. G. Davies, D. Simons; CMMID COVID-19 working group, Effectiveness of isolation, testing, contact tracing, and physical distancing on reducing transmission of SARS-CoV-2 in different settings: A mathematical modelling study. Lancet Infect. Dis. 20, 1151-1160 (2020). doi:10.1016/S1473-3099(20)30457-6 Medline
20. L. Ferretti, C. Wymant, M. Kendall, L. Zhao, A. Nurtay, L. Abeler-Dörner, M. Parker D. Bonsall, C. Fraser, Quantifying SARS-CoV-2 transmission suggests epidemic control with digital contact tracing. Science 368, eabb6936 (2020). doi:10.1126/science.abb6936 Medline

21. R. Hinch, W. J. M. Probert, A. Nurtay, M. Kendall, C. Wymatt, M. Hall, K. Lythgoe, A. B. Cruz, L. Zhao, A. Stewart, L. Ferritti, D. Montero, J. Warren, N. Mather, M. Abueg, N. Wu, A. Finkelstein, D. G. Bonsall, L. Abeler-Dorner, C. Fraser, OpenABM-Covid19 - an agent-based model for non-pharmaceutical interventions against COVID-19 including contact tracing. medRxiv (2020), doi:10.1101/2020.09.16.20195925.

22. A. Barrat, C. Cattuto, M. Kivelä, S. Lehmann, J. Saramäki, Effect of manual and digital contact tracing on COVID-19 outbreaks: a study on empirical contact data. medRxiv (2020), doi:10.1101/2020.07.24.20159947.

23. M. Mancastroppa, C. Castellano, A. Vezzani, R. Burioni, Stochastic sampling effects favor manual over digital contact tracing. arXiv:2010.03399 [cond-mat, physics:physics, q-bio] (2020) (available at https://arxiv.org/abs/2010.03399).

24. L. Lorch, H. Kremer, W. Trouleau, S. Tsirtsis, A. Szanto, B. Schölkopf, M. GomezRodriguez, Quantifying the Effects of Contact Tracing, Testing, and Containment Measures in the Presence of Infection Hotspots. arXiv:2004.07641 [physics, q-bio, stat] (2020) (available at https://arxiv.org/abs/2004.07641).

25. M. E. Kretzschmar, G. Rozhnova, M. C. J. Bootsma, M. van Boven, J. H. H. M. van de Wijgert, M. J. M. Bonten, Impact of delays on effectiveness of contact tracing strategies for COVID-19: A modelling study. Lancet Public Health 5, e452-e459 (2020). doi:10.1016/S2468-2667(20)30157-2 Medline

26. M. Abueg, R. Hinch, N. Wu, L. Liu, W. J. M. Probert, A. Wu, P. Eastham, Y. Shafi, M. Rosencrantz, M. Dikovsky, Z. Cheng, A. Nurtay, L. Abeler-Dörner, D. G. Bonsall, M. V. McConnell, S. O'Banion, C. Fraser, Modeling the combined effect of digital exposure notification and non-pharmaceutical interventions on the COVID-19 epidemic in Washington state. medRxiv (2020), doi:10.1101/2020.08.29.20184135

27. M. Ajelli, B. Gonçalves, D. Balcan, V. Colizza, H. Hu, J. J. Ramasco, S. Merler, A. Vespignani, Comparing large-scale computational approaches to epidemic modeling: Agent-based versus structured metapopulation models. BMC Infect. Dis. 10, 190 (2010). doi:10.1186/1471-2334-10-190 Medline

28. D. L. Chao, M. E. Halloran, V. J. Obenchain, I. M. Longini Jr., T. E. Flu, FluTE, a publicly available stochastic influenza epidemic simulation model. PLOS Comput. Biol. 6, e1000656 (2010). doi:10.1371/journal.pcbi.1000656 Medline

29. Baromètre du numérique 2019 (2019), (available at https://www.credoc.fr/publications/barometre-du-numerique-2019).

30. L. Fumanelli, M. Ajelli, P. Manfredi, A. Vespignani, S. Merler, Inferring the structure of social contacts from demographic data in the analysis of infectious diseases spread. PLOS Comput. Biol. 8, e1002673 doi:10.1371/journal.pcbi.1002673 Medline

31. Q.-H. Liu, M. Ajelli, A. Aleta, S. Merler, Y. Moreno, A. Vespignani, Measurability of the epidemic reproduction number in data-driven contact networks. Proc. Natl. Acad. Sci. U.S.A. 115, 12680-12685 (2018). doi:10.1073/pnas.1811115115 Medline

32. D. Mistry, M. Litvinova, A. Pastore Y Piontti, M. Chinazzi, L. Fumanelli, M. F. C. Gomes, S. A. Haque, Q.-H. Liu, K. Mu, X. Xiong, M. E. Halloran, I. M. Longini Jr., S. Merler, M. Ajelli, A. Vespignani, Inferring high-resolution human mixing patterns for disease modeling. Nat. Commun. 12, 323 (2021). doi:10.1038/s41467-02020544-y Medline

33. G. Béraud, S. Kazmercziak, P. Beutels, D. Levy-Bruhl, X. Lenne, N. Mielcarek, Y Yazdanpanah, P.-Y. Boëlle, N. Hens, B. Dervaux, The French Connection: The First Large Population-Based Contact Survey in France Relevant for the Spread of Infectious Diseases. PLOS ONE 10, e0133203 (2015). doi:10.1371/journal.pone.0133203 Medline

34. Q. Li, X. Guan, P. Wu, X. Wang, L. Zhou, Y. Tong, R. Ren, K. S. M. Leung, E. H. Y. Lau, J. Y. Wong, X. Xing, N. Xiang, Y. Wu, C. Li, Q. Chen, D. Li, T. Liu, J. Zhao, M. Liu, W. Tu, C. Chen, L. Jin, R. Yang, Q. Wang, S. Zhou, R. Wang, H. Liu, Y. Luo, Y. Liu, G. Shao, H. Li, Z. Tao, Y. Yang, Z. Deng, B. Liu, Z. Ma, Y. Zhang, G. Shi, T. T. Y. Lam, J. T. Wu, G. F. Gao, B. J. Cowling, B. Yang, G. M. Leung, Z. Feng, Early Transmission Dynamics in Wuhan, China, of Novel Coronavirus-Infected Pneumonia. N. Engl. J. Med. 382, 1199-1207 (2020). doi:10.1056/NEJMoa2001316 Medline

35. X. He, E. H. Y. Lau, P. Wu, X. Deng, J. Wang, X. Hao, Y. C. Lau, J. Y. Wong, Y. Guan, X. Tan, X. Mo, Y. Chen, B. Liao, W. Chen, F. Hu, Q. Zhang, M. Zhong, Y. Wu, L. Zhao, F. Zhang, B. J. Cowling, F. Li, G. M. Leung, Temporal dynamics in viral shedding 
and transmissibility of COVID-19. Nat. Med. 26, 1-4 (2020). doi:10.1038/s41591020-0869-5

36. H. Salje, C. Tran Kiem, N. Lefrancq, N. Courtejoie, P. Bosetti, J. Paireau, A. Andronico, N. Hozé, J. Richet, C.-L. Dubost, Y. Le Strat, J. Lessler, D. Levy-Bruhl, A. Fontanet, L. Opatowski, P.-Y. Boelle, S. Cauchemez, Estimating the burden of SARS-CoV-2 in France. Science 369, 208-211 (2020). doi:10.1126/science.abc3517 Medline

37. D. Cereda, M. Tirani, F. Rovida, V. Demicheli, M. Ajelli, P. Poletti, F. Trentini, G. Guzzetta, V. Marziano, A. Barone, M. Magoni, S. Deandrea, G. Diurno, M. Lombardo, M. Faccini, A. Pan, R. Bruno, E. Pariani, G. Grasselli, A. Piatti, M. Gramegna, F. Baldanti, A. Melegaro, S. Merler, The early phase of the COVID-19 outbreak in Lombardy, Italy. arXiv:2003.09320 [q-bio] (2020) (available at https://arxiv.org/abs/2003.09320).

38. mbevand, mbevand/florida-covid19-line-list-data

(2020; https:/github.com/mbevand/florida-covid19-line-list-data).

39. Stay-at-home requirements during the COVID-19 pandemic. Our World in Data, (available at https://ourworldindata.org/grapher/stay-at-home-covid).

40. SPF, COVID-19: point épidémiologique du 19 novembre 2020, (available at /maladies-et-traumatismes/maladies-et-infections-respiratoires/infection-acoronavirus/documents/bulletin-national/covid-19-point-epidemiologique-du19-novembre-2020).

41. G. Pullano, L. Di Domenico, C. E. Sabbatini, E. Valdano, C. Turbelin, M. Debin, C. Guerrisi, C. Kengne-Kuetche, C. Souty, T. Hanslik, T. Blanchon, P.-Y. Boëlle, J. Figoni, S. Vaux, C. Campèse, S. Bernard-Stoecklin, V. Colizza, Underdetection of COVID-19 cases in France threatens epidemic control. Nature, 1-9 (2020). Medline

42. G. Pullano, E. Valdano, N. Scarpa, S. Rubrichi, V. Colizza, Evaluating the effect of demographic factors, socioeconomic factors, and risk aversion on mobility during the COVID-19 epidemic in France under lockdown: A population-based study. The Lancet Digital Health 2, e638-e649 (2020). doi:10.1016/S2589-7500(20)30243$\underline{0}$ Medline

43. R. Li, S. Pei, B. Chen, Y. Song, T. Zhang, W. Yang, J. Shaman, Substantial undocumented infection facilitates the rapid dissemination of novel coronavirus (SARS-CoV-2). Science 368, 489-493 (2020). doi:10.1126/science.abb3221 Medline

44. Coronavirus (COVID-19) Testing - Statistics and Research. Our World in Data, (available at https://ourworldindata.org/coronavirus-testing).

45. Réseau Sentinelles $>$ France $>$ Accueil, (available at https://websenti.u707.jussieu.fr/sentiweb/).

46. L. E. Smith, H. W. W. Potts, R. Amlot, N. T. Fear, S. Michie, J. Rubin, Adherence to the test, trace and isolate system: results from a time series of 21 nationally representative surveys in the UK (the COVID-19 Rapid Survey of Adherence to Interventions and Responses [CORSAIR] study). medRxiv (2020), doi:10.1101/2020.09.15.20191957.

47. K. Chang, A University Had a Great Coronavirus Plan, but Students Partied On. The New York Times (2020), (available at https:/www.nytimes.com/2020/09/10/health/university-illinois-covid.html).

48. L. Atlani-Duault, B. Lina, D. Malvy, Y. Yazdanpanah, F. Chauvin, J.-F. Delfraissy, COVID-19: France grapples with the pragmatics of isolation. Lancet Public Health 5, e573-e574 (2020). doi:10.1016/S2468-2667(20)30235-8 Medline

49. TousAntiCovid sur Twitter. Twitter, (available at https://twitter.com/TousAntiCovid/status/1328272121177452551).

50. Immuni - I numeri di Immuni, (available at www.immuni.italia.it).

51. The curve of people downloading COVIDSafe has flattened. Has the app done enough? (2020), (available at https://www.abc.net.au/news/2020-0602/coronavirus-covid19-covidsafe-app-how-many-downloads-greghunt/12295130).

52. Nearly $40 \%$ of Icelanders are using a covid app-and it hasn't helped much. MIT Technology Review, (available at https://www.technologyreview.com/2020/05/11/1001541/iceland-rakningc19-covid-contact-tracing/).

53. I. Montagni, N. Roussel, R. Thiébaut, C. Tzourio, The French Covid-19 contact tracing app: Knowledge, attitudes, beliefs and practices of students in the health domain. J. Med. Internet Res. (2021). doi:10.2196/26399

54. DP-3T/documents (DP^3T, 2020; https:/github.com/DP-3T/documents).
55. M. Nanni, G. Andrienko, A.-L. Barabási, C. Boldrini, F. Bonchi, C. Cattuto, F. Chiaromonte, G. Comandé, M. Conti, M. Coté, F. Dignum, V. Dignum, J. DomingoFerrer, P. Ferragina, F. Giannotti, R. Guidotti, D. Helbing, K. Kaski, J. Kertesz, S. Lehmann, B. Lepri, P. Lukowicz, S. Matwin, D. M. Jiménez, A. Monreale, K. Morik, N. Oliver, A. Passarella, A. Passerini, D. Pedreschi, A. Pentland, F. Pianesi, F. Pratesi, S. Rinzivillo, S. Ruggieri, A. Siebes, V. Torra, R. Trasarti, J. V. D. Hoven, A. Vespignani, Give more data, awareness and control to individual citizens, and they will help COVID-19 containment. Ethics Inf. Technol. 1-6 (2021). doi:10.1007/s10676-020-09572-w Medline

56. I. Z. Kiss, D. M. Green, R. R. Kao, Infectious disease control using contact tracing in random and scale-free networks. J. R. Soc. Interface 3, 55-62 (2006). doi:10.1098/rsif.2005.0079 Medline

57. J. Stehlé, N. Voirin, A. Barrat, C. Cattuto, L. Isella, J.-F. Pinton, M. Quaggiotto, W. Van den Broeck, C. Régis, B. Lina, P. Vanhems, High-resolution measurements of face-to-face contact patterns in a primary school. PLOS ONE 6, e23176 (2011). doi:10.1371/journal.pone.0023176 Medline

58. M. Génois, A. Barrat, Can co-location be used as a proxy for face-to-face contacts? EPJ Data Sci. 7, 1-18 (2018). doi:10.1140/epids/s13688-018-0140-1

59. D. J. A. Toth, M. Leecaster, W. B. P. Pettey, A. V. Gundlapalli, H. Gao, J. J. Rainey, A. Uzicanin, M. H. Samore, The role of heterogeneity in contact timing and duration in network models of influenza spread in schools. J. R. Soc. Interface 12 20150279 (2015). doi:10.1098/rsif.2015.0279 Medline

60. H. Hartle, F. Papadopoulos, D. Krioukov, Dynamic Hidden-Variable Network Models, arXiv:2101.00414 [physics] (2021) (available at https://arxiv.org/abs/2101.00414).

61. M. Boguñá, R. Pastor-Satorras, Class of correlated random networks with hidden variables. Phys. Rev. E Stat. Nonlin. Soft Matter Phys. 68, 036112 (2003). doi:10.1103/PhysRevE.68.036112 Medline

62. G. Laurent, J. Saramäki, M. Karsai, From calls to communities: A model for timevarying social networks. Eur. Phys. J. B 88, 301 (2015). doi:10.1140/epjb/e2015 $\underline{60481-x}$

\section{ACKNOWLEDGMENTS}

Funding: This study was partially supported by the ANR project DATAREDUX (ANR19-CE46-0008-01) to AB, VC and PYB; EU H2O20 grants MOOD (H2O2O874850) to VC, CP, and PYB, and RECOVER (H2020-101003589) to VC; the Municipality of Paris (https://www.paris.fr/) through the program Emergence(s) to JAML, BAG, PB, FP and CP; the ANR and Fondation de France through the project NoCOV (00105995) to PYB, CP; the Spanish Ministry of Science and Innovation to JAML and BAG, the AEl and FEDER (EU) under the grant PACSS (RTI2018-093732-B-C22) JAML and BAG; the Maria de Maeztu program for Units of Excellence in R\&D (MDM-2017-0711) to JAML and BAG.Author contributions: CP conceived the study: PYB, VC, AB, CP designed the study and interpreted the results; $\mathrm{CP}$, wrote the original draft; PB, LB, FP implemented the synthetic population model; JAML, BAG, implemented the epidemic model; All authors edited the manuscript and approved its final version. Competing interests: All authors declare that they have no competing interests. Data and materials availability: All data needed to evaluate the conclusions in the paper are present in the paper or available from public sources cited on the paper.

\section{SUPPLEMENTARY MATERIALS}

advances.sciencemag.org/cgi/content/full/sciadv.abd8750/DC1

Submitted 18 July 2020

Accepted 22 February 2021

Published First Release 12 March 2021

$10.1126 /$ sciadv.abd8750 
A

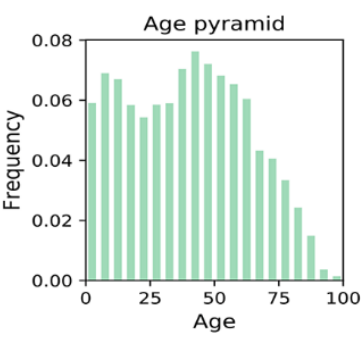

B

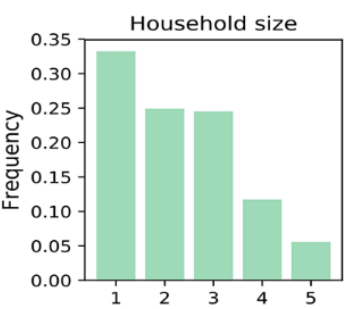

C

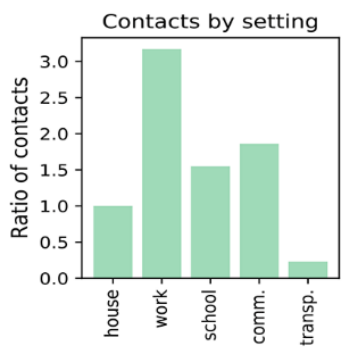

D

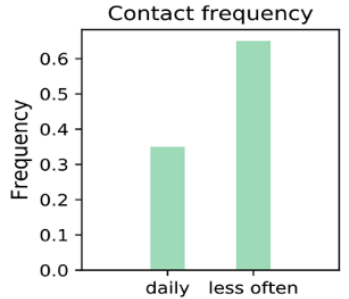

$\mathbf{E}$

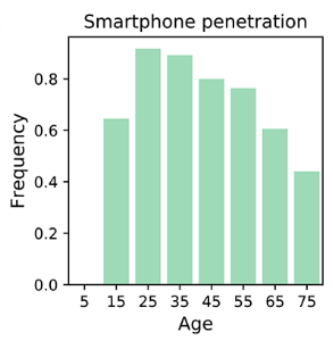

$\mathbf{H}$

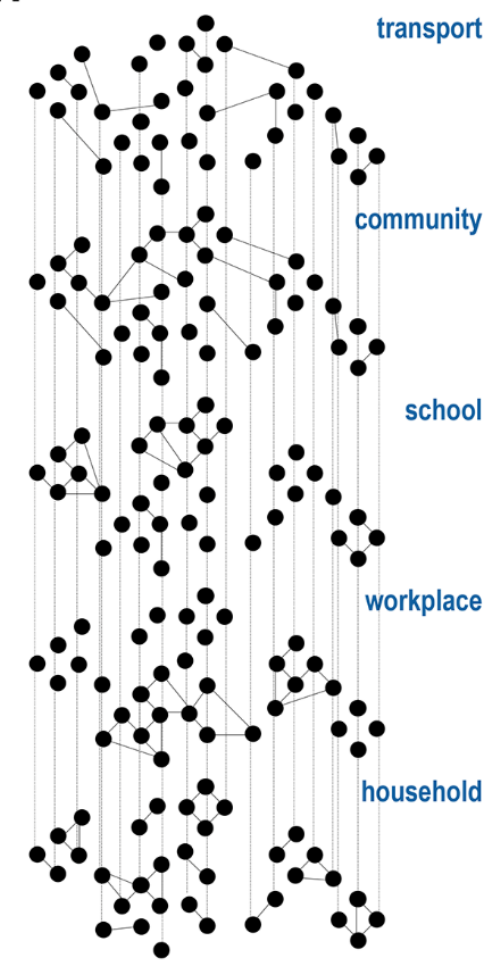

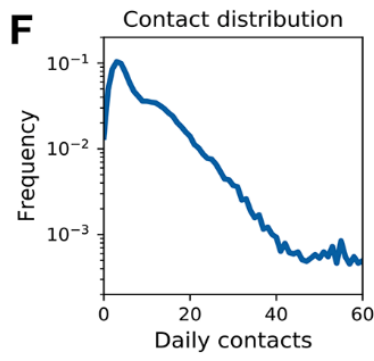

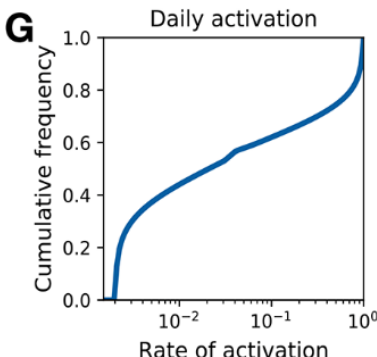

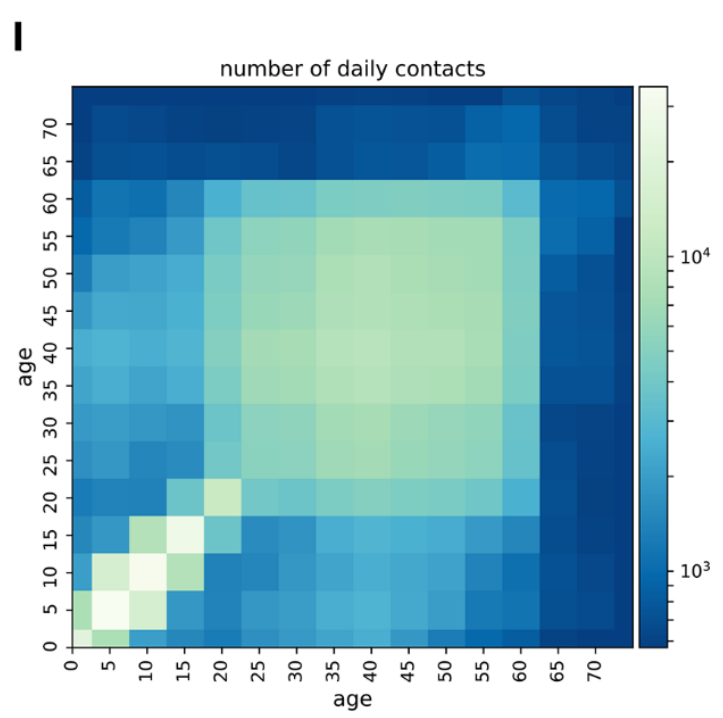

Fig. 1. Synthetic population. A-E Key statistics used as input for the synthetic population reconstruction. A Age pyramid for France (source INSEE). B Household size (source INSEE). C Ratio of contacts by setting with respect to household contacts (33). D Fraction of contacts occurring each day or less frequently (33). E Smartphone penetration by age. The overall average adoption in the population is 64\% (29). F Distribution of the number of daily contacts in the model. G Cumulative distribution of the activation rate associated to the contacts in the model, calibrated in order to be consistent with the information of panel D. H Sketch of the construction of the contact network: contacts among individuals were represented as a multi-layer dynamical network, where each layer includes contacts occurring in a specific setting. I Age contact matrix computed from the contact network model. 
A

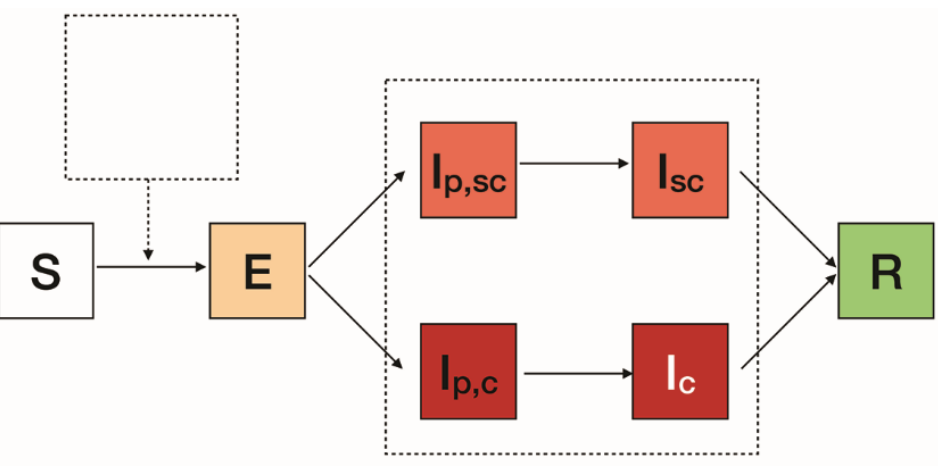

B
transition
transition rate
$S \rightarrow E$
$\beta \sigma_{A} \beta_{I} \omega_{s}$
$E \rightarrow I_{p, s c}$
$\epsilon p_{s c}^{A}$
$E \rightarrow I_{p, c}$
$\epsilon\left(1-p_{s c}^{A}\right)$
$I_{p, s c} \rightarrow I_{c}, I_{p, c} \rightarrow I_{c}$
$\mu_{p}$
$I_{s c}, I_{c} \rightarrow R$
$\mu$
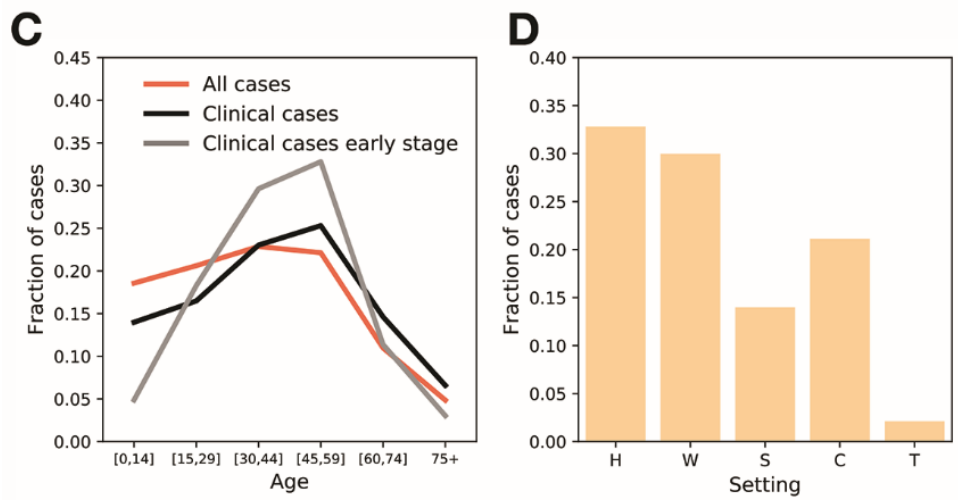

Fig. 2. Modelling COVID-19 epidemic. A, B Compartmental model summarizing the epidemic states and transitions between states. Parameters and their values are reported in Table 1. C Cases by age for an uncontrolled epidemic. We show all cases (clinical and subclinical) in red and clinical cases in black. The grey line shows the clinical cases in the early stage of the epidemic (here defined as the first 30 days), with less cases among children than in later stages. D Transmission by setting $(\mathrm{H}, \mathrm{W}, \mathrm{S}, \mathrm{C}, \mathrm{T}$ stand respectively for household, workplace, school, community, transport). The simulations were done with $\beta=0.25$ corresponding to $R_{0}=3.1$. Additional aspects of the outbreak are reported in the Supplementary Material. 

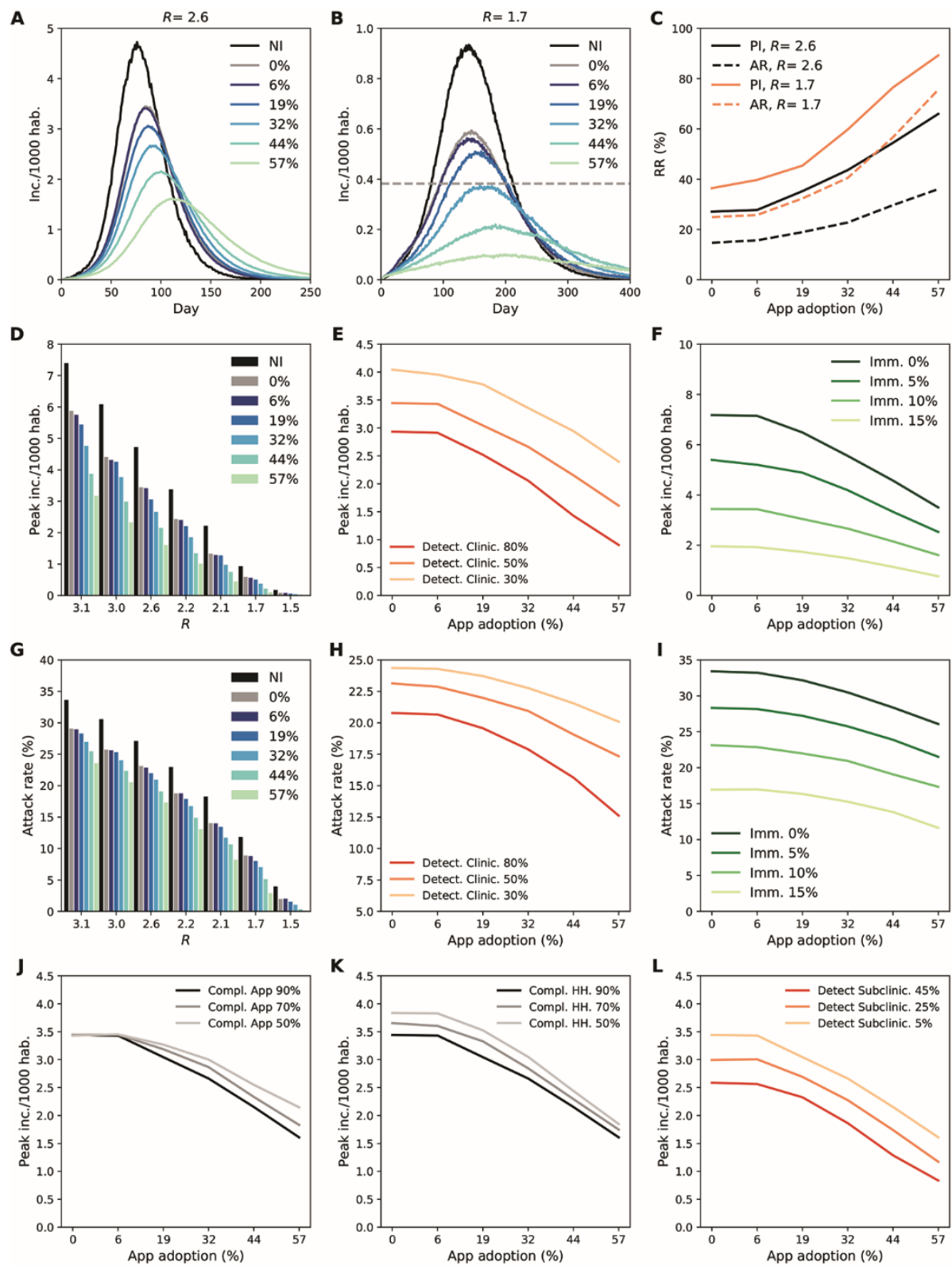

Fig. 3. Impact of digital contact tracing and household isolation on the epidemic. A, B Incidence (clinical cases) according to app adoption for $R=2.6$ and $R=1.7$, respectively. The black curve shows the scenario with no intervention ( $\mathrm{NI}$ ). Other curves correspond to app adoption levels ranging from $0 \%$ (household isolation only) to $57 \%$ (90\% of smartphone users). Incidence threshold level corresponding to ICU saturation is showed as a dashed grey line in panel B. C Relative reduction ( $R R$ ) in attack rate (AR) and peak incidence (PI) as a function of the app adoption for the scenarii shown in A and B. $R R$ is computed as $\frac{x_{\text {ref }}-x}{x_{\text {ref }}}$, where $x$ is either PI or AR and $x_{\text {ref }}$ is the value of the quantity with no intervention. Attack rate is computed as cumulative incidence discounting initial immunity (10\%). D, G Peak incidence and attack rate according to reproduction ratio $R$ and app adoption. E, H Peak incidence and attack rate according to app adoption and percentage of clinical cases detected. F, I Peak incidence and attack rate according to app adoption and initial immunity. J Peak incidence according to app adoption and compliance to isolation of contacts notified by the app. K Peak incidence according to app adoption and compliance to isolation of household contacts. L Peak incidence according to app adoption and percentage of subclinical cases detected. Except as otherwise indicated, parameters values were: initial immunity $10 \%$, clinical case detection $50 \%$, subclinical case detection $5 \%$, compliance to isolation of contacts notified by the app $90 \%$, compliance to isolation of household contacts $90 \%, R=2.6$.

First release: 12 March 2021 
A
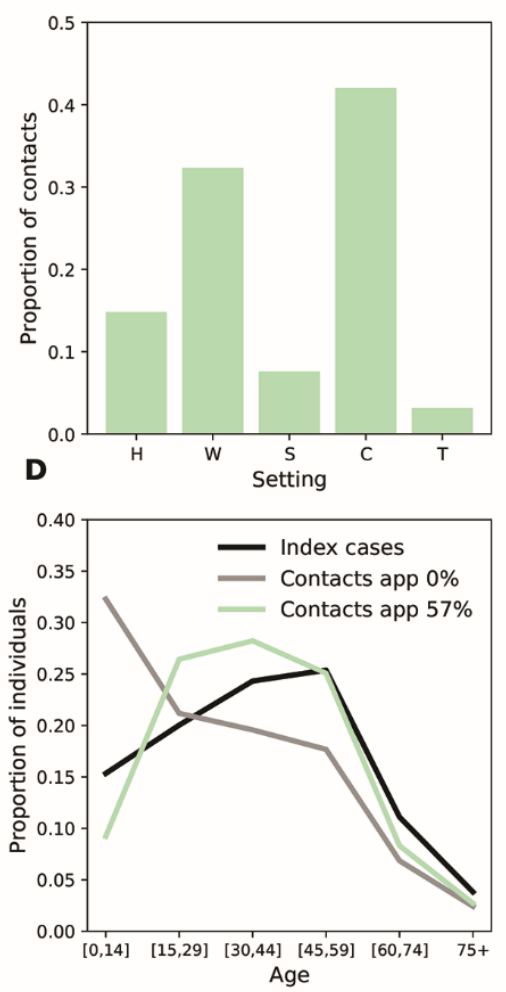

B
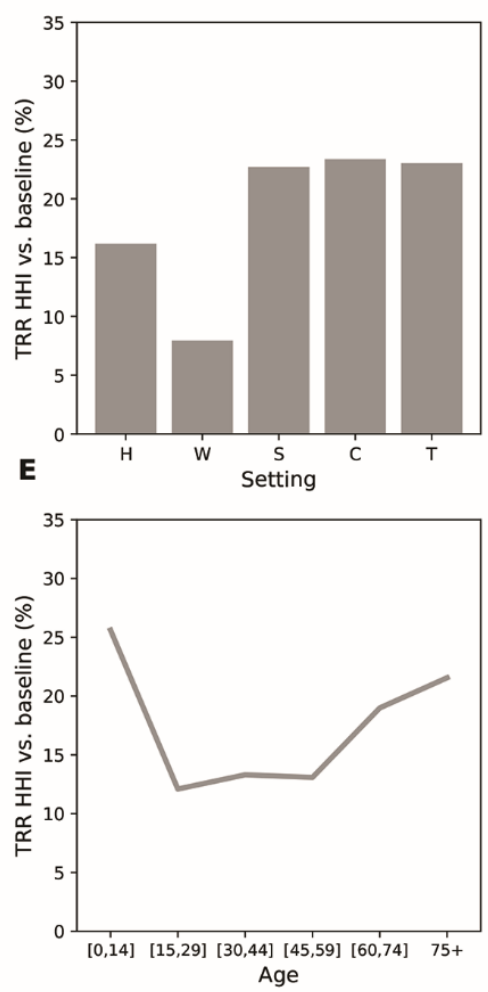

C
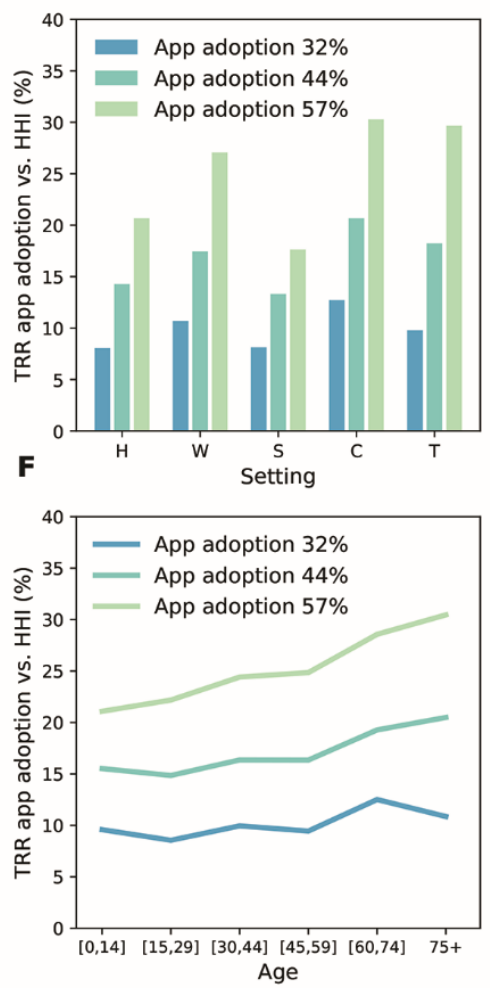

Fig. 4. Effect of digital contact tracing and household isolation by age and setting. A Repartition among the different settings of the contacts detected by contact tracing (57\% app adoption). B Relative reduction in transmission (TRR) by setting obtained with household isolation. The relative reduction in transmission is here defined as $\operatorname{TRR}(s)=\frac{I_{\text {ref }}^{s}-I^{s}}{I_{\text {ref }}^{s}}$, where $I^{s}$ is the total number of clinical and subclinical cases infected in setting $s$, in the given intervention scenario considered (here household isolation) and $I_{\text {ref }}^{s}$ is the same quantity in the reference scenario (here the scenario with no intervention). $\mathrm{C}$ $T R R$ obtained with digital contact tracing with respect to household isolation only, for three values of app adoption. D Repartition among the different age groups of the index cases and of the detected contacts, in a scenario with household isolation only, and with the inclusion of digital contact tracing (57\% app adoption). The repartition of index cases is very similar in the two scenarios, thus only the one with household isolation is shown for the sake of clarity. $\mathrm{E}$ TRR by age group of the infected as obtained with household isolation only. F TRR of digital contact tracing with respect to household isolation only. We assume $R=2.6$, immunity $10 \%$ and probability of detection $50 \%$. 
A

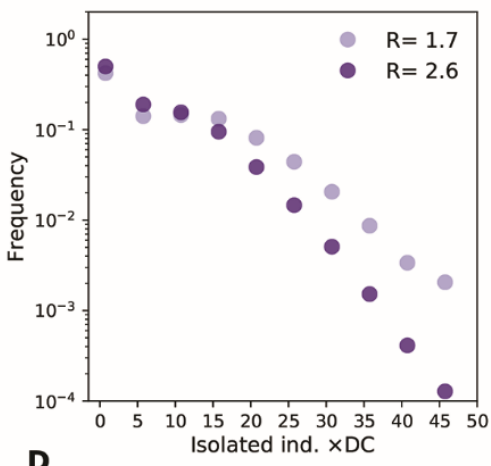

D

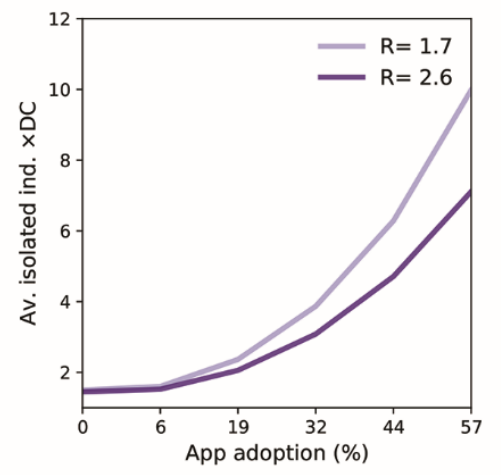

B

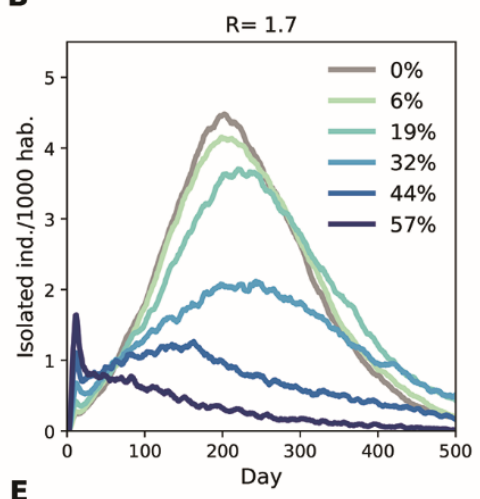

$\mathbf{E}$

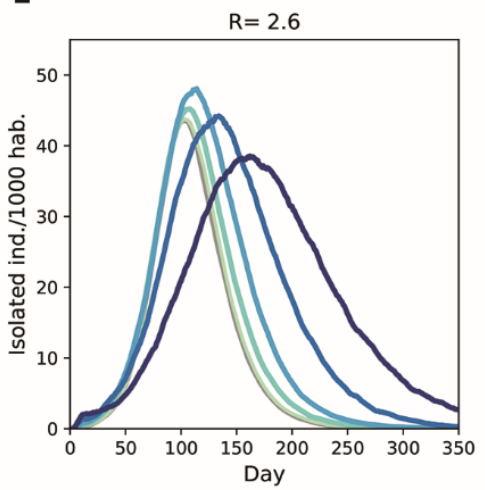

C
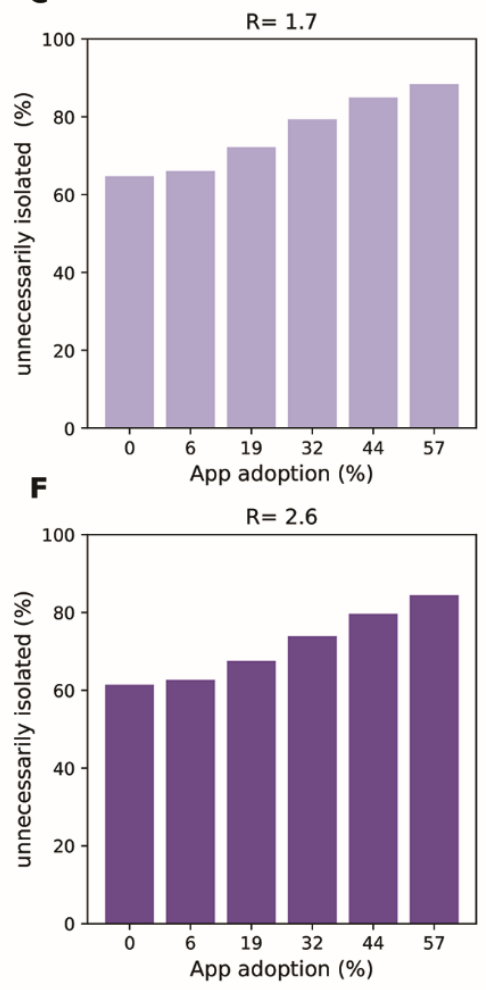

Fig. 5. Impact of combined digital contact tracing and household isolation on the isolation of individuals. A Distribution of the number of isolated individuals per detected case (DC) for $57 \%$ of app adoption. D Average number of isolated individuals per detected case as a function of app adoption. $\mathrm{B}$, E Percentage of the population isolated as a function of time for $R=1.7$ (B) $R=2.6$ (E). C, F: Fraction of unnecessary isolated, i.e., fraction of contacts isolated without being positive. 
Table 1. Compartmental model parameters and their values

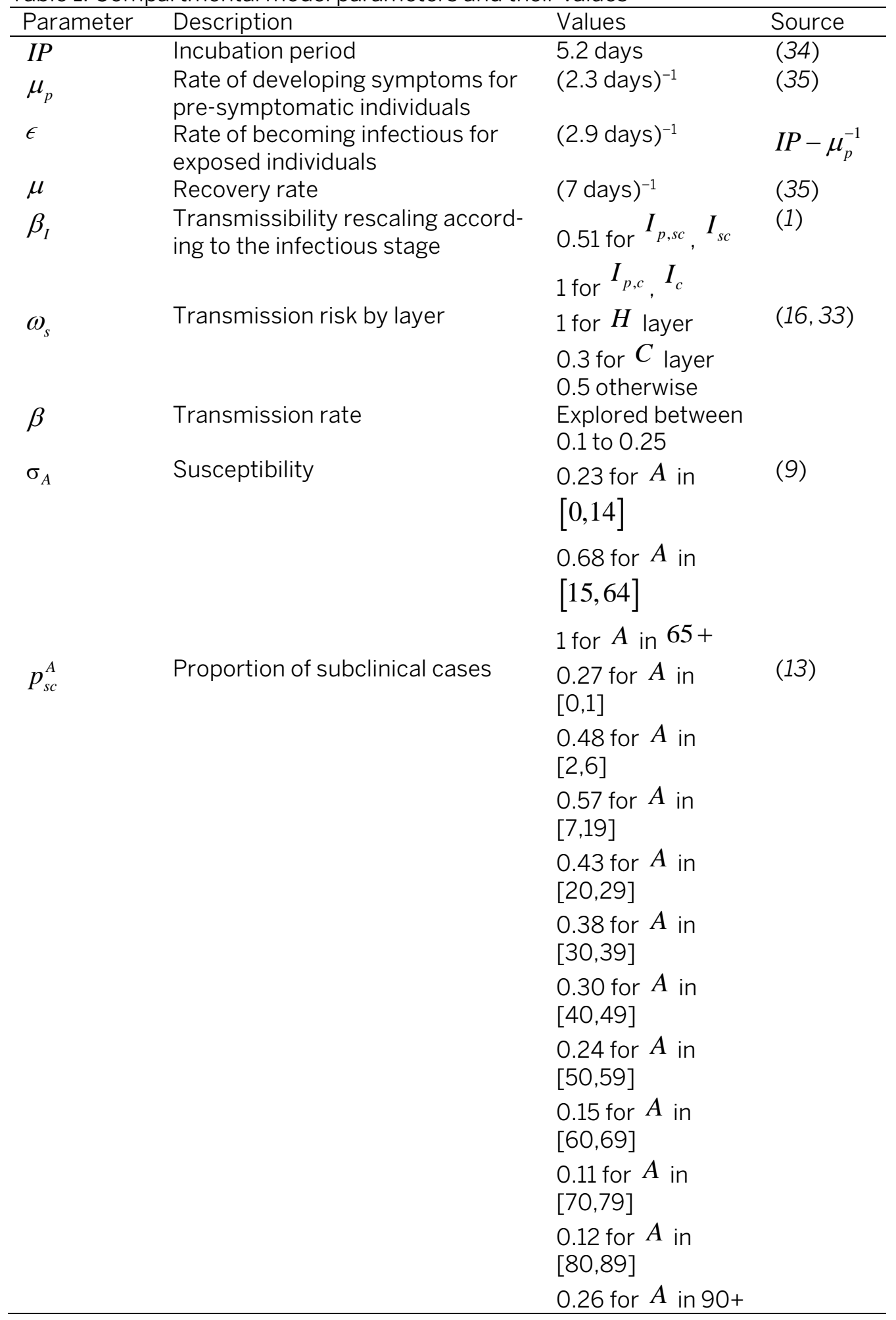

